\title{
A Novel Small Molecule Therapy for Corneal Neovascularization
}

\author{
Zachary Barry ${ }^{1}$, Bomina Park ${ }^{1}$, Timothy W. Corson ${ }^{1}$ \\ ${ }^{1}$ Indiana University School of Medicine, Department of Ophthalmology
}

Background and Hypothesis: Corneal neovascularization (CNV) is characterized by abnormal blood vessel growth in the avascular cornea. CNV can be caused by multiple insults such as trauma, infection, and immunological diseases. Current treatments have partial efficacy and associated side effects, revealing a need for novel treatments.

We identified the enzyme soluble epoxide hydrolase (sEH) as a target of an antiangiogenic small molecule we developed, $\mathrm{SH}-11037$. SH-11037 was antiangiogenic in mouse models for retinal and choroidal neovascularization. However, its effect on CNV has not been explored. We hypothesize that inhibition of sEH with $\mathrm{SH}-11037$ will block CNV.

Experimental Design or Project Methods: To validate sEH's relevance in CNV, we assessed the effects of inhibition of sEH in an alkali burn CNV mouse model, treating the eyes with either eyedrops of $\mathrm{SH}-11037$ or another known sEH inhibitor, t-AUCB. Neovascularization was determined by clinical assessment and immunohistochemistry. Toxicity in the retina was assessed by optical coherence tomography (OCT). Eyedrops of dexamethasone $(0.1 \%$ and $0.03 \%)$ were positive controls.

Results: Clinical assessment indicated that there was a strong trend towards a decrease in CNV in the $0.1 \%$ dexamethasone control group $(p=0.0509)$. Further data generation is underway, but we expect to see decreased CNV in the SH11037 and t-AUCB experimental groups, compared to the vehicle (PBS) control group.

Conclusion and Potential Impact: Upon completion of this study there is potential for a new therapeutic target for reducing CNV. This study provides the assessment of sEH's involvement in CNV, which opens future areas of study. 